\title{
Spin-orbit misalignment in the HD 80606 planetary system ${ }^{\star} \star \star \star$
}

\author{
F. Pont ${ }^{1}$, G. Hébrard ${ }^{2}$, J. M. Irwin ${ }^{3}$, F. Bouchy ${ }^{2,4}$, C. Moutou ${ }^{5}$, D. Ehrenreich ${ }^{6}$, T. Guillot ${ }^{7}$, S. Aigrain ${ }^{1}$, X. Bonfils ${ }^{6}$, \\ Z. Berta ${ }^{4}$, I. Boisse ${ }^{2}$, C. Burke ${ }^{10}$, D. Charbonneau ${ }^{4}$, X. Delfosse ${ }^{6}$, M. Desort ${ }^{6}$, A. Eggenberger ${ }^{6}$, T. Forveille ${ }^{6}$, \\ A.-M. Lagrange ${ }^{6}$, C. Lovis $^{8}$, P. Nutzman ${ }^{3}$, F. Pepe ${ }^{8}$, C. Perrier ${ }^{6}$, D. Queloz $^{8}$, N. C. Santos ${ }^{9}$, D. Ségransan ${ }^{8}$, \\ S. Udry ${ }^{8}$, and A. Vidal-Madjar ${ }^{2}$
}

\author{
1 School of Physics, University of Exeter, Exeter EX4 4QL, UK \\ e-mail: fpont@astro.ex.ac.uk; fredericjpont@gmail.com \\ 2 Institut d'Astrophysique de Paris, UMR7095 CNRS, Université Pierre \& Marie Curie, 98bis boulevard Arago, 75014 Paris, France \\ 3 Harvard-Smithsonian Center for Astrophysics, 60 Garden Street, Cambridge, MA 02138, USA \\ 4 Observatoire de Haute-Provence, 04870 Saint-Michel l'Observatoire, France \\ 5 Laboratoire d'Astrophysique de Marseille, UMR 6110, CNRS \& Univ. de Provence, 38 rue Frédéric Joliot-Curie, \\ 13388 Marseille Cedex 13, France \\ ${ }^{6}$ Laboratoire d'Astrophysique, Observatoire de Grenoble, Université J. Fourier, BP 53, 38041 Grenoble Cedex 9, France \\ 7 Université de Nice-Sophia Antipolis, Observatoire de la Côte d'Azur, CNRS UMR 6202, BP 4229, 06304 Nice Cedex 4, France \\ 8 Observatoire de Genève, Université de Genève, 51 Chemin des Maillettes, 1290 Sauverny, Switzerland \\ 9 Centro de Astrofísica, Universidade do Porto, Rua das Estrelas, 4150-762 Porto, Portugal \\ 10 Space Telescope Science Institute, 3700 San Martin Dr., Baltimore, MD21218, USA
}

Received 11 May 2009 / Accepted 30 June 2009

\section{ABSTRACT}

\begin{abstract}
We recently reported the photometric and spectroscopic detection of the primary transit of the 111-day-period, eccentric extra-solar planet HD 80606b, at Observatoire de Haute-Provence, France. The whole egress of the primary transit and a section of its central part were observed, allowing the measurement of the planetary radius, and evidence for a spin-orbit misalignment through the observation of the Rossiter-McLaughlin anomaly. The ingress not having been observed for this long-duration transit, uncertainties remained in the parameters of the system. We present here a refined, combined analysis of our photometric and spectroscopic data, together with further published radial velocities, ground-based photometry, and Spitzer photometry around the secondary eclipse, as well as new photometric measurements of HD 80606 acquired at Mount Hopkins, Arizona, just before the beginning of the primary transit. Although the transit is not detected in those new data, they provide an upper limit for the transit duration, which narrows down the possible behaviour of the Rossiter-McLaughlin anomaly in the unobserved part of the transit. We analyse the whole data with a Bayesian approach using a Markov-chain Monte Carlo integration on all available information. We find $R_{\mathrm{p}}=0.98 \pm 0.03 R_{\mathrm{Jup}}$ for the planetary radius, and a total primary transit duration of $11.9 \pm 1.3 \mathrm{~h}$ from first to fourth contact. Our analysis reinforces the hypothesis of spin-orbit misalignment in this system (alignment excluded at $>95 \%$ level), with a positive projected angle between the planetary orbital axis and the stellar rotation (median solution $\lambda \sim 50^{\circ}$ ). As HD 80606 is a component of a binary system, the peculiar orbit of its planet could result from a Kozai mechanism.
\end{abstract}

Key words. stars: planetary systems

\section{Introduction}

HD 80606 is a solar-type star with a gas giant planetary companion on a highly eccentric 111-day orbit (Naef et al. 2001). With $e=0.93$, the planet receives about a thousand times more star light at periastron than at apastron, which makes it a key system to study the atmospheric and thermal properties of hot gas giant planets. By a lucky coincidence (about 1 percent probability for a randomly oriented orbit), the orbital plane is aligned with the line-of-sight, so that both the secondary eclipse and

\footnotetext{
* Based on observations made with the 1.20-m and 1.93-m telescopes at Observatoire de Haute-Provence (CNRS), France, by the SOPHIE consortium (program 07A.PNP.CONS), and with a 16-inch telescope at Mt. Hopkins, Arizona, USA, by the MEarth team.

$\star \star$ Full table of photometric measurements is only available in electronic form at the CDS via anonymous ftp to

cdsarc.u-strasbg.fr $(130.79 .128 .5)$ or via

http://cdsweb.u-strasbg.fr/cgi-bin/qcat?J/A+A/502/695
}

primary transit were detected. The secondary eclipse was measured during a long photometric run with the Spitzer space telescope (Laughlin et al. 2009). In Moutou et al. (2009, hereafter M09), we presented our detection of the primary transit, simultaneously measured in photometry and spectroscopy with the 1.2-m and 1.93-m telescopes at Observatoire de Haute-Provence, France. The spectroscopic transit data seemed to indicate that the orbital plane of the planet was not aligned with the stellar rotation axis. But since the transit ingress was not observed, a large degree of uncertainty remained in this parameter, as well as in the latitude of the transit and radius of the host star.

Photometric data of the same event are available from two other teams and locations. Fossey et al. (2009, F09) presented data obtained at the Mill Hill London Observatory, England, with two telescopes (a 35-cm Celestron and a 25-cm Meade), on a time span that almost matches that of our OHP observations the same night: data were obtained during the main portion of the flat part of the transit, the whole egress, and a few 
hours after its end. Garcia-Melendo \& McCullough (2009, G09) presented data obtained with a $60-\mathrm{cm}$ telescope at the Esteve Duran Observatory, Spain, on a shorter time span: the observations started just before the egress. No detection of the transit ingress has been reported at the time of writing.

In this paper, we present photometric data of HD 80606 taken from Mt Hopkins, Arizona on the same night with the MEarth network (Irwin et al. 2009; Nutzman \& Charbonneau 2008). These data show no flux variation, but they do provide a powerful constraint on the system parameters by imposing a strict lower limit to the beginning of the transit. We apply a Bayesian analysis to the whole data set, together with previous radialvelocity monitoring and the Spitzer observations near secondary eclipse, to calculate accurate values of the system parameters, including the radii of the host star and planet, and the spin-orbit angle.

\section{Observations}

\subsection{MEarth photometry}

A single field containing HD 80606 and HD 80607 was monitored continuously using one telescope of the MEarth observatory located at the Fred Lawrence Whipple Observatory on Mount Hopkins, Arizona, for the night of 2009 February 13. Additional observations were taken on 2009 February 14th and 15 th but these are not used in the present work. MEarth uses a non-standard $715 \mathrm{~nm}$ long-pass filter, with the response limited at the red end by the long-wavelength tail of the CCD quantum efficiency curve.

Observations were started at the end of nautical twilight (solar elevation $12^{\circ}$ below the horizon), and continued until the start of nautical twilight in the morning. The airmass of the field varied from 1.9 at the start of observations, to a minimum of 1.1 at meridian transit, which occurred at UT 07:11, and at the end of observations was 2.5. A total of $16954.8 \mathrm{~s}$ exposures were obtained at an average cadence of $\sim 23 \mathrm{~s}$ including overheads (CCD readout time, and re-centering the field after each exposure).

Due to the extremely short exposures necessary to avoid saturation, and the small telescope aperture of $0.4 \mathrm{~m}$, errors on individual measurements are dominated by atmospheric scintillation. It was not possible to defocus the telescope due to the small $\left(\sim 21^{\prime \prime}\right)$ separation between HD 80606 and HD 80607. We use the formula of Young (1967) to estimate the contribution to our observational error bars arising from scintillation. As noted by Ryan \& Sandler (1998), the typical coherence length for this effect is $\sim 12^{\prime \prime}$, so the standard formula should remain a reasonable approximation.

Data were reduced using the standard MEarth reduction pipeline, which is at present largely identical to the Monitor project pipeline described in Irwin et al. (2007). An aperture radius of 10 pixels (corresponding to 7 ". 6 on-sky) was used to extract differential photometry. In order to estimate the contamination in the aperture for one star from the other given this large aperture size, we use measurements in multiple concentric apertures from single-stars in the same field as our target to derive a simple curve-of-growth. From this we estimate that $<0.3 \%$ of the flux from HD 80607 falls inside the aperture centered on HD 80606, and vice versa. This is negligible for our purposes, since it would lead to a $<1 \%$ underestimation in transit depths, which is smaller than the observational error in this quantity.

We used HD 80607 as a comparison star to derive differential light curves of HD 80606 from this photometry. Since these stars have similar positions on the detector and almost
Table 1. Photometric times series for HD 80606 from MEarth (full table available electronically).

\begin{tabular}{lll}
\hline \hline Date [HJD] & $F_{715}[\mathrm{mag}]$ & $\sigma_{F}$ \\
\hline 2454875.589851 & 8.116900 & 0.005537 \\
2454875.590117 & 8.133620 & 0.005522 \\
2454875.590395 & 8.142982 & 0.005507 \\
2454875.590684 & 8.160600 & 0.005493 \\
2454875.590950 & 8.126864 & 0.005480 \\
2454875.591217 & 8.142918 & 0.005471 \\
2454875.591494 & 8.129089 & 0.005450 \\
2454875.591749 & 8.153709 & 0.005435 \\
2454875.592015 & 8.074484 & 0.005425 \\
$\ldots$ &.. & $\ldots$ \\
\hline
\end{tabular}

identical colors, effects such as color-dependent atmospheric scintillation and flat fielding error are minimized by doing this, and we find no advantage to attempting to use other stars of comparable brightness on the field as additional comparison stars. MEarth uses German Equatorial Mounts, so the entire telescope and detector system must be rotated through $180^{\circ}$ relative to the sky upon crossing the meridian. Using HD 80607 as a comparison star, we see little evidence for flat fielding errors in the data for HD 80606, which normally manifest as different base-line levels in the light curve for positive and negative hour angle. We therefore apply no correction for this effect in the present analysis.

The MEarth data is given in Table 1.

\subsection{OHP 120-cm photometry}

The photometric observations of HD 80606 and HD 80607 performed at the 120-cm telescope at OHP during the nights 2009 February 12 and 13 were presented by M09. The transit was detected during the second night, which is the only one of the two that we use in the present work. 326 frames were secured during the transit night, with 20 to 30 -s exposure times. The negative slope after egress tends to suggest that a correction for airmass variations should be taken into account in deriving the flux. The airmass is 1.5 at the beginning of the night, then reaches 1.0 at the middle of the egress, and increases up to 1.66 at the end of the night. Assuming that the slope seen out of transit is due to airmass changes, and that the effect of airmass on the photometry is linear, we find a correction of $(2-$ airmass $) \times 0.004$. The impact of the correction is to create a small slope at the beginning of the transit sequence, i.e. during the flat section of the transit. The significance of this new slope is low. Since this correction dominates the error budget on the transit parameters, it is important to take correlated noise into account in the analysis.

\subsection{SOPHIE Doppler spectroscopy}

Radial velocities of HD 80606 measured with the SOPHIE spectrograph at the 1.93-m telescope of OHP were presented by M09. A continuous sequence of 39 measurements was acquired during the transit night (2009, 13-14 February), as well as nine extra measurements between 8th and 18th February, out of the transit. The radial velocities were obtained from a weighted cross-correlation of the spectra with a G2-type numerical template. All the spectra present a similar signal-to-noise ratio, $S / N \simeq 47$ per pixel at $550 \mathrm{~nm}$. Together with the parameters of the cross-correlation function of the spectra (full width at half maximum of $7.33 \pm 0.02 \mathrm{~km} \mathrm{~s}^{-1}$, and a contrast representing 
$48.2 \pm 0.3 \%$ of the continuum), this corresponds to a $\sim 2.4 \mathrm{~m} \mathrm{~s}^{-1}$ photon-noise uncertainty on the radial velocity measurements. We quadratically added $3.5 \mathrm{~m} \mathrm{~s}^{-1}$ due to telescope guiding errors and $1 \mathrm{~m} \mathrm{~s}^{-1}$ due to wavelength calibration, resulting in radial velocities with a typical accuracy of $\sim 4.5 \mathrm{~m} \mathrm{~s}^{-1}$.

\subsection{Published Doppler and photometric data}

We complemented our SOPHIE measurements with published radial velocities from three other instruments: 74 measurements from ELODIE at OHP (Naef et al. 2001; Moutou et al. 2009), 46 HIRES measurements from Keck (Butler et al. 2006), and $23 H R S$ measurements from the Hobby-Eberly Telescope (Wittenmyer et al. 2009). The typical accuracies of these three extra datasets are $12.3,5.0$, and $8.3 \mathrm{~m} \mathrm{~s}^{-1}$ respectively, and their time spans are Nov. 1999-Nov. 2003, Apr. 2001-Feb. 2005, and Dec. 2004-Mar. 2007, respectively. None of those measurements were obtained during a primary transit. These measurements are used to refined the Keplerian orbit of the planet. Systematic radial velocity shifts between the different datasets are unknown, and left as free parameters. The residuals around the model orbit show that the ELODIE uncertainties are underestimated, and we scaled these uncertainties upwards by a factor 1.8 in order to obtain coherent normalized residuals.

The egress of the transit of 14th February 2009 was observed in photometry from three locations covering part of the transit center and the transit egress (M09, F09, G09; see Sect. 1).

HD 80606 was also monitored for nearly $24 \mathrm{~h}$ around the time of the secondary eclipse at 8 microns with the Spitzer space telescope (Laughlin et al. 2009). These data provide strong additional constraints on the system parameters by measuring the time and duration of the secondary eclipse.

We include these data sets in our combined solution (we use only the Celestron time series from F09).

\section{Analysis}

Figures 1, 2 and 3 display the photometric and spectroscopic data for the radial velocity curve, the spectroscopic transit and the photometric transit. The secondary eclipse is plotted in Laughlin et al. (2009). Figure 4 shows the configuration of the system according to the best-fit solution in Sect. 4 .

The procedures to infer physical parameters from observations of transiting planetary systems have now been firmly established, and numerous descriptions can be found in the recent literature (see e.g. contributions to Pont et al. 2009, for reviews and examples). The case of HD 80606b requires all the toolbox of the trade for several reasons: the extreme eccentricity makes the relation between physical parameters and observable quantities even more non-linear than usual, the incomplete coverage of the transit in photometry and spectroscopy requires sound Bayesian statistics, most data sets are dominated by correlated noise rather than random errors, and different types of information (transit and secondary eclipse photometry, radial velocity orbit, stellar evolution models) provide partial constraints of comparable importance. For these reasons, we need to use a fully Bayesian approach, with physically meaningful priors on all parameters. We also need proper accounting for correlated noise.

Although the data is incomplete in some respects (absence of detection of the transit ingress), the abundant datasets leave little leeway for most parameters. In particular, the combination of the secondary eclipse duration, the egress duration and the orbital parameters tightly constrain the mass and size of the host star and the planet.
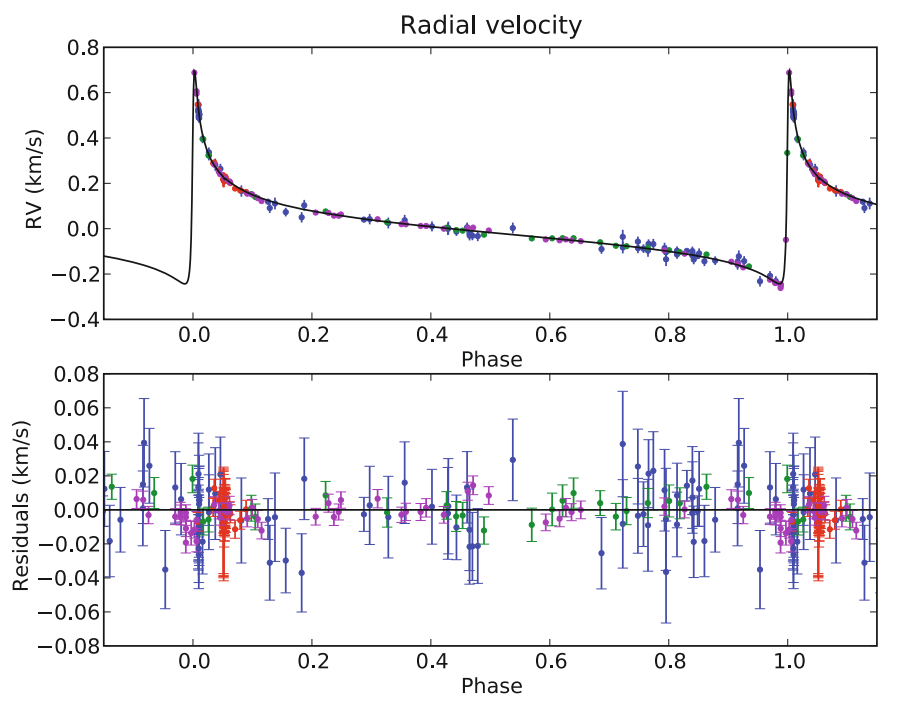

Fig. 1. Radial-velocity data and model. Data from Elodie (blue), Sophie (red), Keck (magenta) and HET (green).

\subsection{Method}

We use a Bayesian approach to determine the probability distribution of the physical parameters given all the available observations and prior information such as stellar evolution models. We perform the integration with a Markov Chain Monte Carlo (MCMC) algorithm, using Metropolis-Hastings sampling. This is similar to the procedure described for instance in Collier Cameron et al. (2007).

This technique is particularly sensitive to the assumptions used on the distribution of measurement uncertainties. MCMC integration samples the merit function used to measure the agreement between model and observations according to the Bayesian prior probability, and as such it cannot be better than the merit function used. If a traditional "observed vs. measured" sum-of-squares is chosen, the error underestimation due to correlated noise that plague straightforward fitting methods will also be observed. To avoid biased results and unrealistically narrow posterior probability distributions for the output parameters, it is essential to use realistic error estimates for all data, including systematic errors. In the photometric and transit spectroscopic time series of HD 80606 the dominant source of systematics are fluctuations correlated in time (e.g. airmass and seeing effects). Pont et al. (2006) discuss the effects of this "red noise" and a possible way to integrate it in the merit function.

\subsection{Correlated noise}

For most datasets pertaining to HD 80606, red (i.e. correlated) noise is by far the dominant source of uncertainties: (1) the SOPHIE coverage of the spectroscopic transit was acquired during a single night. It is known that in such conditions, SOPHIE data are sensitive to weather-related systematics at the level of a few meters-per-second; (2) the photometric times series also cover only the transit egress. When only a partial transit is observed with ground-based photometry, the correction of slow trends is difficult and dominates the error budget; (3) the out-oftransit baseline flux of the Spitzer observations is made variable at the $10^{-4}$ level by instrumental effects and by the light reflected on the day side of the planet, a factor which, from the point of view of parameter determination, is equivalent to red noise. 


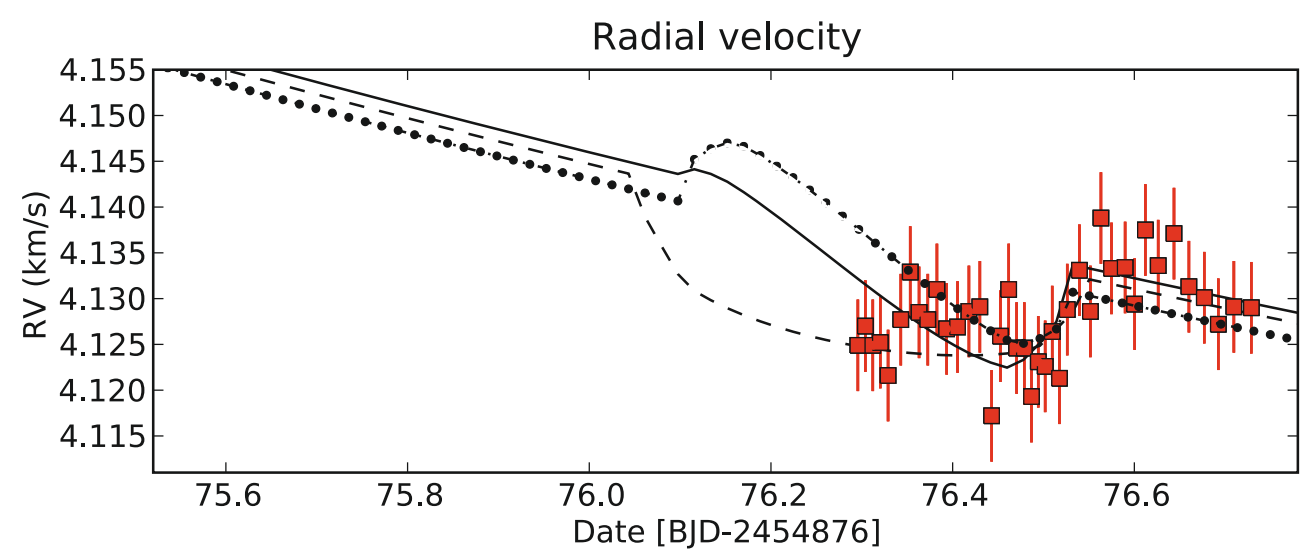

Fig. 2. Radial-velocity data and models around the phase of transit. The three model curves correspond to spin-orbit angles at the best-fit solution $\left(\lambda=33^{\circ}\right.$, solid line), a good solution with a higher spin-orbit angle $\left(\lambda=122^{0}\right.$, dashed), and the highest-likelihood solution with aligned spin and orbit $(\lambda=0$, dash-dotted).

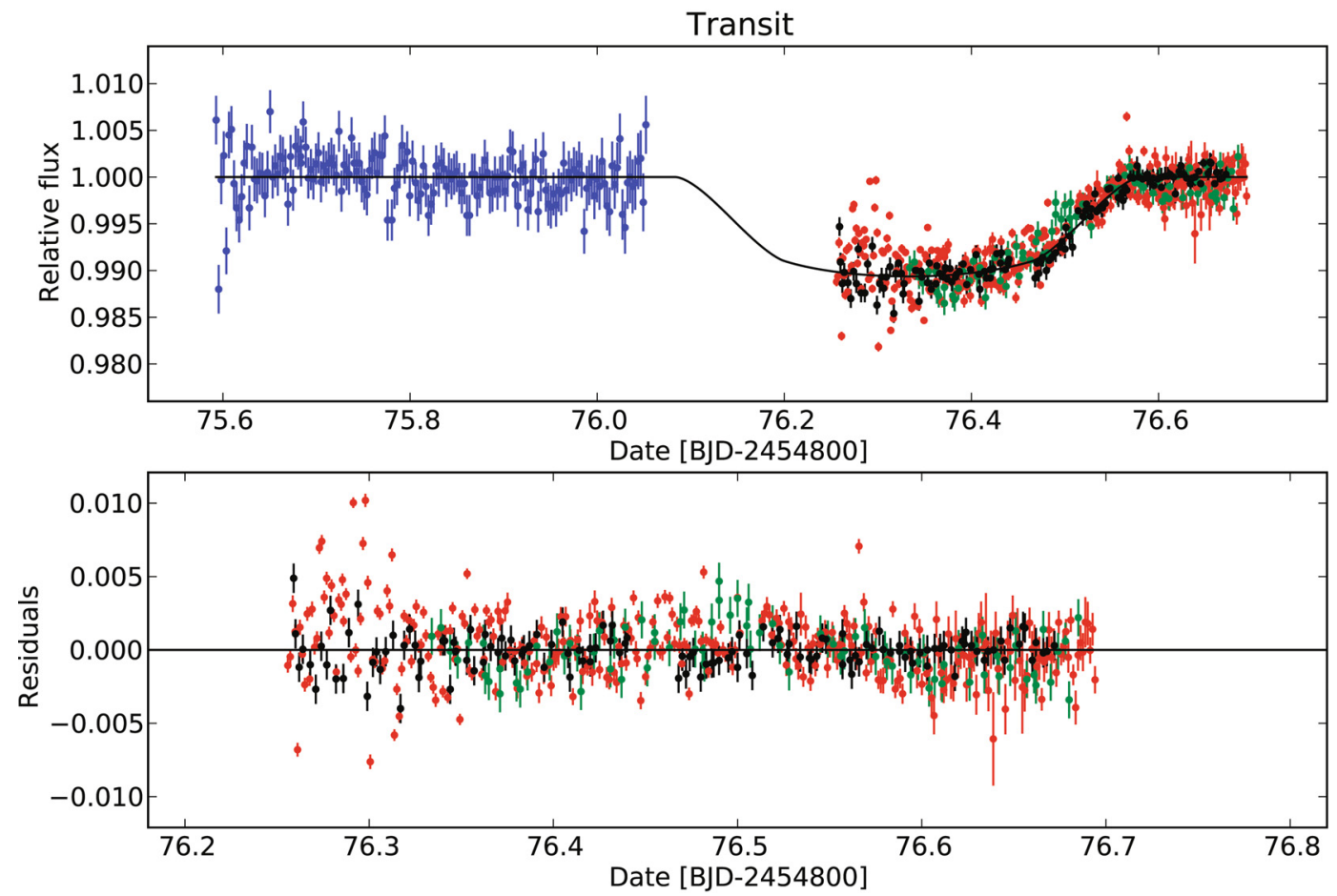

Fig. 3. Photometric data on 13-14 Feb. 2009 transit. Red: M09, black: MEarth, blue: F09, green: G09.

We model the red noise as described in Pont et al. (2006), with a single $\sigma_{\text {r }}$ parameters for each data set, describing the amplitude of correlated noise over the relevant timescale. For the photometric time series we use the " $V(n)$ " method described in that paper, which estimates $\sigma_{\mathrm{r}}$ from the departure of binned versions of the data compared to purely white noise. We find $\sigma_{\mathrm{r}}=7 \times 10^{-4}$ for the OHP photometry, $8 \times 10^{-4}$ for the MEarth photometry, $3.6 \times 10^{-4}$ for the F09 photometry, $8 \times 10^{-4}$ for the G09 photometry, $4 \mathrm{~m} \mathrm{~s}^{-1}$ for the Sophie transit spectroscopy and $2 \times 10^{-4}$ for the Spitzer time series. The first three values are coherent with our experience of ground-based transit spectroscopy, with $\sigma_{\mathrm{r}}$ between 4 and $10 \times 10^{-4}$ being typical for single-object rapid cadence time series. The value of the correlation parameter for the SOPHIE time series is also compatible with previous experience (e.g. Hébrard et al. 2008). Finally, there is enough outof-transit data in the Spitzer time series to measure $\sigma_{\mathrm{r}}$ precisely. Our red noise analysis thus indicates that the F09 Celestron data is subject to lower systematics than the M09 and G09 photometric measurements (as visible as well in the behaviour of the residuals compared to model lightcurves). This implies that the first data set will get about twice as much weight as each of the other two in the combined analysis. To account for the clearly much lower quality of the photometric data at the beginning and end of the night, we increase $\sigma_{\mathrm{r}}$ by a factor three for these measurements $(J D<2454876.34)$.

\subsection{Models}

We use the Mandel \& Agol (2002) algorithm to build model transit and secondary eclipse lightcurves, with a linear limbdarkening law with $u=0.66$ (suitable for a solar-type star; at this point the accuracy of the data does not require higher-order modelling of the limb darkening). We use the Ohta et al. (2005) analytical description of the RM effect, and a Keplerian radialvelocity orbit. We compute the $3 \mathrm{D}$ position of the planet relative to the star at each date. Two additional parameters are introduced 


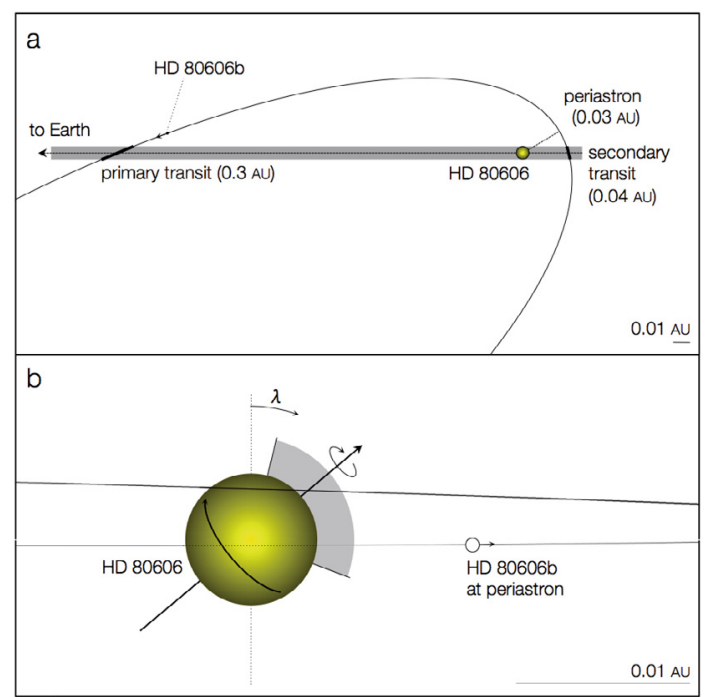

Fig. 4. Geometry of the HD 80606 system according to our best-fit solution, a) from above the orbit, b) seen from Earth.

to describe the photometric signal of the secondary eclipse: a planet-to-star surface-brightness ratio in the Spitzer band, and an out-of-transit continuum flux. The brightness variation of the planet around the time of secondary eclipse is neglected.

\section{4. $M C M C$}

We use a chain with 200000 steps, starting with the parameters in M09. The size of the steps is set to 0.05 times the uncertainties quoted by that study. The free parameters are the following: $P, T_{0}, i, e, \varpi, V_{0}^{1 . .4}, M_{\mathrm{pl}}, R_{\mathrm{pl}}, M_{\mathrm{s}}, R_{\mathrm{s}}, d T, \lambda, V_{\text {rot }}$, respectively the period, epoch of periastron, orbital inclination, orbital eccentricity, argument of periastron, center-of-mass radial velocity (for each spectrograph independently), planet mass and radius, star mass and radius, planet-to-star surface brightness ratio in the Spitzer band, spin-orbit angle and projected stellar spin. We use Gibbs sampling for the steps in the chain (next step accepted with a probability equal to the ratio of likelihoods). We include the constraints from stellar evolution models directly in the prior and in the merit function used in the MCMC: the MCMC chain moves in an interpolation of the Girardi et al. (2002) stellar evolution models with uniform steps in mass, age and metallicity (corresponding to a flat prior in these quantities). Each evolution model produces values for the stellar mass, radius and temperature, $M_{\mathrm{s}}, R_{\mathrm{s}}$ and $T_{\text {eff }}$, that are used to estimate the merit function. How this method solves Bayes' theorem is studied in more details in the context of stellar ages in Pont \& Eyer (2004).

\subsection{Merit functions}

The merit function in the MCMC is taken as the likelihood of the observations given the model, assuming gaussian error distributions and purely "white+red" noise (Pont et al. 2006), using all the photometric and radial velocity data, as well as the spectroscopically determined stellar parameters:

$$
\begin{aligned}
\chi^{2}=\sum_{\text {phot }} \frac{\left(F_{\mathrm{obs}}-F_{\mathrm{mod}}\right)^{2}}{\sigma_{F w}^{2}+N \sigma_{F r}^{2}} & +\sum_{\mathrm{rv}} \frac{\left(V_{\mathrm{obs}}-V_{\mathrm{mod}}\right)^{2}}{\sigma_{V w}^{2}+N \sigma_{V r}^{2}} \\
& +\sum_{\mathrm{star}} \frac{\left(S_{\mathrm{obs}}-S_{\mathrm{mod}}\right)^{2}}{\sigma_{S}^{2}}
\end{aligned}
$$

where $F$ are the photometric data, $V$ the radial velocity data, $S$ the stellar parameters, and the obs and mod subscripts denote observed and model values respectively. The $w$ and $r$ subscript indicate white and red noise parameters, and $N$ is the number of data points during the correlation timescale. $F_{\text {mod }}$ is the model light curve for the transit and secondary eclipse, $V_{\text {mod }}$ the model radial velocity curve including the RM anomaly during transit, and $S_{\text {mod }}$ the set of observable parameters of the host star according to the Girardi et al. (2002) models. The sums are made respectively on the individual photometric observations, spectroscopic observations, and input stellar parameters of the evolution models (age, mass and metallicity). We take the duration of the egress as the relevant correlation timescale to evaluate $N$ $(\sim 25$ points for photometric time series, 5 points for the radial velocity sequence).

\subsection{Prior distributions}

We define the steps in the MCMC corresponding to flat prior distributions in the following quantities: $P, T_{0}, \cos i, V_{i}, e \cos \varpi$, $e \sin \varpi, K \sqrt{1-\mathrm{e}^{2}}, R_{p} / R_{\mathrm{s}}, M_{\mathrm{s}}, \tau_{\mathrm{s}},[\mathrm{Fe} / \mathrm{H}], d T, \cos \lambda$. The combinations were chosen either for physical reason (isotropic orientation of spin and orbit in space, random epoch), to avoid strong covariance in the MCMC (radius ratio instead of radius, eccentricity dependence of $K$ integrated in the prior) or linear when the prior is not significant compared to the observational constraints $\left(P, V_{i}\right)$.

The prior distribution in the parameters of the parent star $\left(M_{\mathrm{s}}, R_{\mathrm{s}}, T_{\text {eff }}\right)$ corresponds to a flat distribution in age, mass and metallicity, as described above. We therefore do not reduce the information from stellar evolution models to a single term in the merit function, but make use of the full information provided by the stellar evolution models, thus taking into account the correlation of stellar mass, radius and temperature, and the respective probability of different models for a field star in the solarneighbourhood.

We set the stellar rotational velocity to $V_{\text {rot }}=1.8 \mathrm{~km} \mathrm{~s}^{-1}$ (Fischer \& Valenti 2005). This parameter influences the shape of the spectroscopic transit radial velocity curve, but is poorly constrained by the current data. The results do not vary significantly if we repeat the procedure with a moderate uncertainty on this value (up to about $1 \mathrm{~km} \mathrm{~s}^{-1}$ ). If this parameter is left completely free, the Markov chain does not converge, because different combinations of the rotation velocity, spin-orbit angle and impact parameter produce similar predictions for the observed portion of the spectroscopic transit.

Altogether, three prior distributions have a significant effect on the solution: the orbital inclination angle (penalizing grazing transits compared to a non-Bayesian fit), the stellar age prior (penalizing rare or inexistant combinations of stellar parameters), and the stellar rotation velocity (lifting the main degeneracy in the spectroscopic transit).

\section{Results and discussion}

Table 2 gives the median values and central $68 \%$ confidence intervals for the parameters of the HD80606 system given by the MCMC integration. Note that because the median values correspond to different individual solutions for each parameter, the orbital parameters in the table do not correspond to the best-fit orbit. The best-fit orbit is $P=111.43605$ days, $K=476.48 \mathrm{~m} \mathrm{~s}^{-1}$, $e=0.9332, T_{0}=2454424.8529 \mathrm{BJD}, \varpi=300.71^{0}$. 
Table 2. Parameters for the HD 80606 system. Uncertainties from the $68 \%$ central probability interval of the posterior distribution traced by the Markov chain.

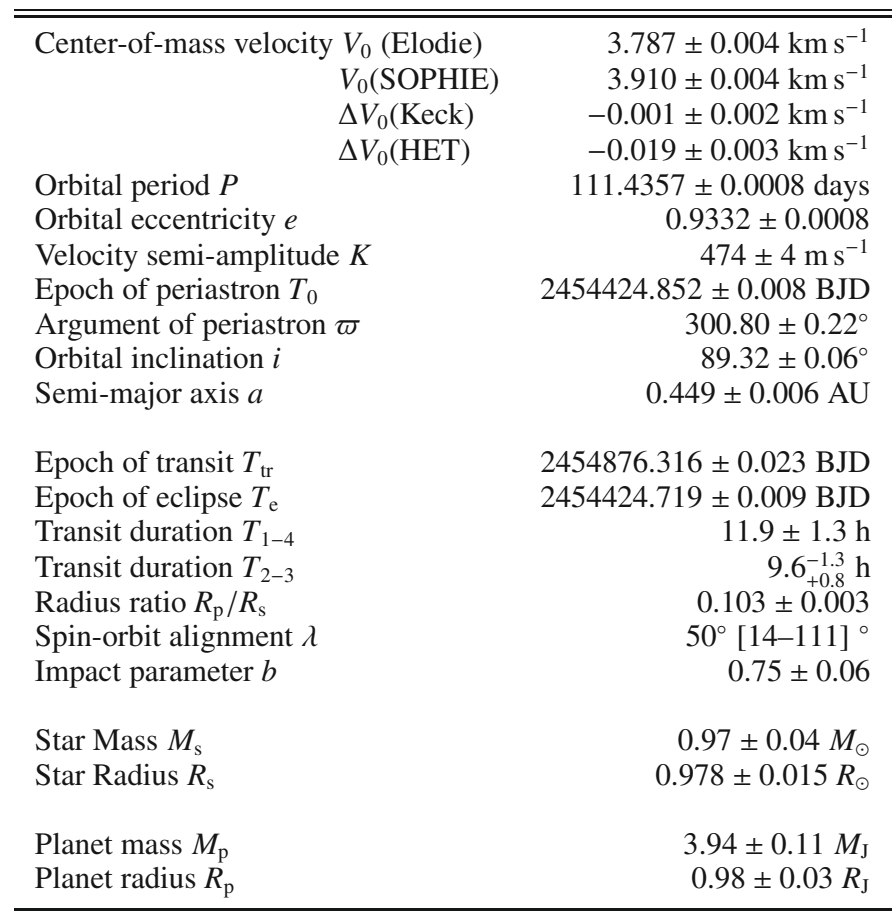

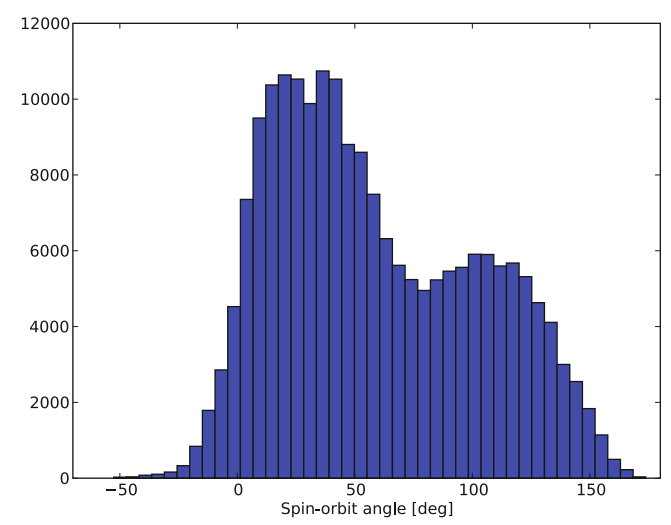

Fig. 5. Posterior probability distribution for the projected spin-orbit angle.

Figures 5 and 6 show the probability distribution functions for the spin-orbit angle and transit duration (first to fourth contact).

The strongest covariance between the output parameters is that of the impact parameter with the transit duration. In general, the transit duration depends mainly on the radius of the host star and impact parameter. When the whole transit is measured, the main covariance is between impact parameter and host star radius. However, in our case, the star radius is relatively well constrained by the duration of the secondary eclipse, and the transit duration is weakly constrained because the transit ingress was not observed, so that the main covariance is between the transit duration and impact parameter (higher impact parameters corresponding to shorter transit). Figure 7 shows the posterior probability density in the $D$ vs. $b$ plane, and in the $D$ vs. $R_{\mathrm{p}}$ plane. The core of the probability distribution in each parameter is well described by Normal distributions, with a tail of

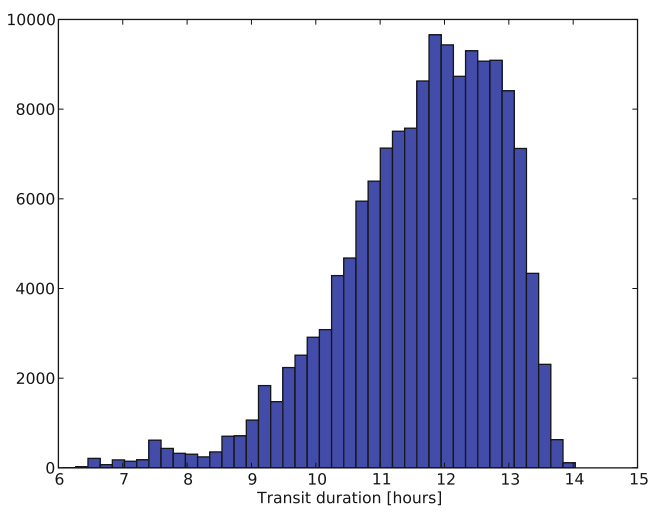

Fig. 6. Posterior probability distribution for the transit duration (first to fourth contact).

low-probability solutions at high impact parameter, low transit duration and larger planet.

Figure 8 shows the probability density in stellar mass and radius. The main correlation is $M \sim R^{3}$, because the transit parameters are degenerate in these quantities. The sharper limit towards larger masses is due to stellar evolution models.

\subsection{Uncertainty intervals}

F09 quote very small uncertainties for the transit duration, orbital inclinations and planetary radius $\left(1.029 \pm 0.017 \mathrm{R}_{\mathrm{J}}, 12.1 \pm\right.$ $0.4 \mathrm{~h}, 89.285 \pm 0.023$ degrees). The uncertainties are small because F09 keep several important parameters fixed (the host star radius and orbital parameters), and purely uncorrelated noise is assumed in the photometry. These simplifications are useful for a rapid initial analysis, but will yield underestimated uncertainties. As extensively discussed in the context of previous observations of transiting planets with high-cadence photometry, the uncertainty on the density $\left(M R^{-3}\right)$ of the primary and the systematics in the photometric noise are actually the dominant sources of uncertainties on most system parameters, including the planetary radius, inclination angle and transit duration. This explains why our uncertainty intervals are larger than those quoted in F09, in spite of being based on a larger ensemble of data.

The larger uncertainties are also due to the fact that the M09, F09 and G09 light curves indicate slightly different shapes for the transit egress (M09 and G09 favour a longer egress, therefore a higher-latitude transit across a larger star). The shape difference between the three lightcurves clearly illustrates that correlated noise dominates the error budget. The presence of correlated noise is also apparent in the SOPHIE sequence, since no parameters for the RM effect can reproduce the sudden jump shortly after the beginning of the data sequence.

For these reasons, as well as the arguments and examples presented in Pont et al. (2006), the probability distributions described by the MCMC integration including correlated noise, uncertainties on all parameters, and host star properties constrained by stellar evolution models, arguably provide a better description of the actual implications of the data in terms of physical parameters and confidence intervals.

\subsection{Spin-orbit angle}

The posterior probability distribution for the projected spin-orbit angle $\lambda$ corresponds to configurations in which the planet crosses mainly the receding limb of the star. An aligned orbit $(\lambda=0)$ is 

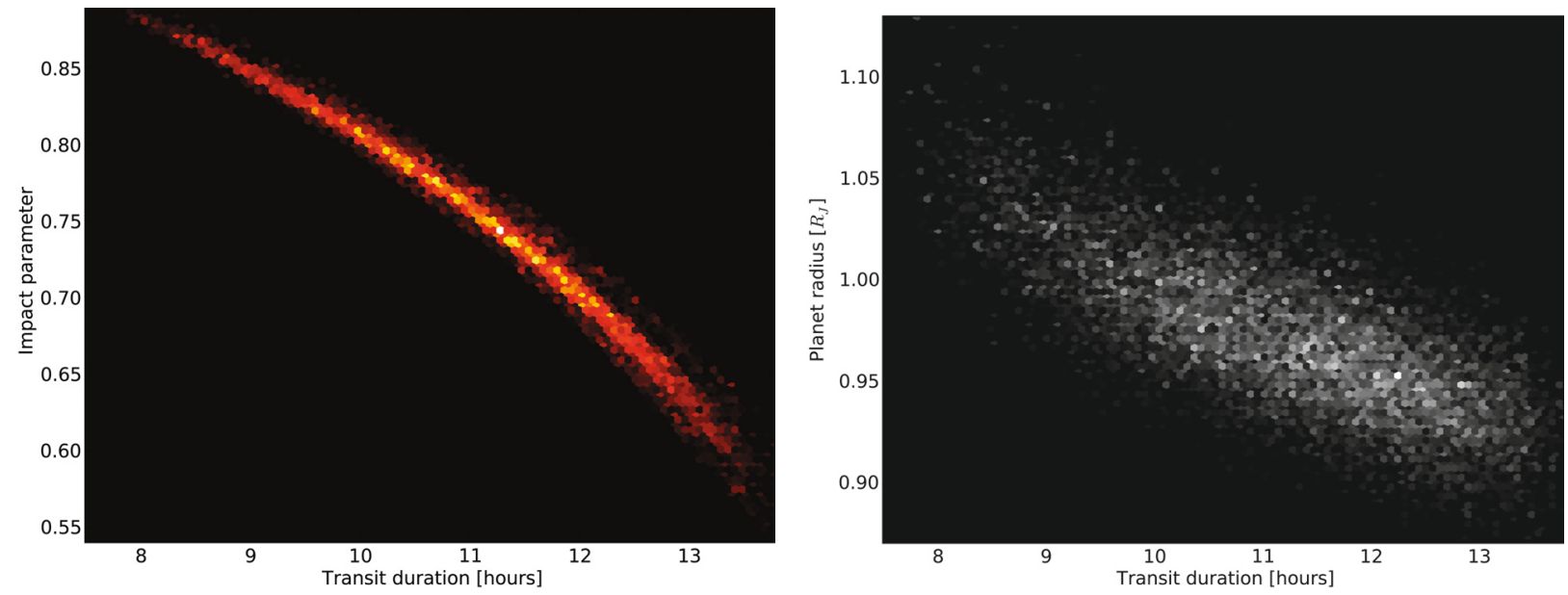

Fig. 7. Posterior probability density: transit duration vs. impact parameter (left) and transit duration vs. planetary radius (right).

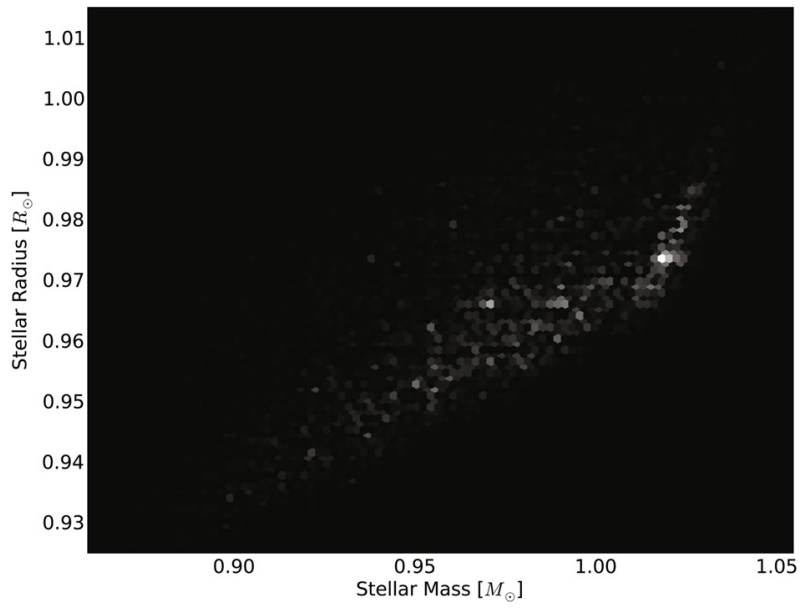

Fig. 8. Posterior probability density: stellar mass and radius.

excluded to a $>95 \%$ level of confidence. The MCMC calculation shows that a spin-orbit alignment in the system is made unlikely by the combination of the shape of the SOPHIE radial velocity time series during transit and the fact that the MEarth photometry excludes a low-latitude transit by placing a strict upper limit on the transit duration.

The result is sensitive to the level of correlated noise assumed in the radial velocity data. This is easily understood: since the amplitude of the RM anomaly is of the order of $15 \mathrm{~m} \mathrm{~s}^{-1}$, assuming systematics of similar amplitude can reconcile the observed shape with that expected in case of spin-orbit alignment by attributing most of the observed variation to systematics. The presence of some systematics in the SOPHIE data is apparent from the mismatch between the first few measurements during transit, to the level of a few $\mathrm{m} \mathrm{s}^{-1}$, but the excellent correspondence between the moment of photometric and spectroscopic egress indicates that the systematics do not dominate the SOPHIE sequence, and therefore that such a high value of $\sigma_{\mathrm{r}}$ is unlikely (note that in our standard solution we already increase the correlated noise for the first measurements in the night to $12 \mathrm{~m} \mathrm{~s}^{-1}$, which is conservative).

Therefore, our conclusion is that the combined data indicate a tilted system, with the planet crossing the receding side of the star during the transit. HD 80606 is the second highly tilted planetary orbit known, after XO-3 (Hébrard et al. 2008; Winn et al. 2009), out of the twelve measured systems
(Fabrycky \& Winn 2009). More strikingly, four of these planets have eccentric orbits, and two of those have tilted orbit. We may therefore be seeing early indications that in addition to having a broad eccentricity distribution, extrasolar gas giants have a bimodal distribution of spin-orbit angles (Fabrycky \& Winn 2009). A possible caveat is that both XO-3 and HD 80606 are high-mass gas giants, which may have formed differently than Jupiter-mass planets (Ribas \& Miralda-Escudé 2007).

\subsection{Planetary radius}

The size of the planet is well constrained by the data, in spite of the fact that only the transit egress was observed. This may seem surprising. It is due to the observation of the secondary eclipse and the peculiarity of the orbit of HD80606b: the planet is much nearer to the star during the secondary eclipse than during the transit, because of the large eccentricity and the position of the periastron. As a result, the mere fact that the planet is transiting implies that the secondary eclipse occurs at low latitude behind the star (see Fig. 4). This lifts the usual degeneracy between stellar radius and impact parameter, and means that the former can be derived reliably from the duration of the secondary eclipse, even in the absence of the constraint from the fractional duration of the ingress/egress. The probability distribution of the stellar radius is further narrowed by the Bayesian prior on the inclination angle, which penalizes high-latitude transits, by the shape of the transit egress, and by stellar evolution models.

The main remaining uncertainty on the size of the planet is due to global systematics in the photometry. The slightly longer egress duration favoured by the M09 and G09 data would indicate a higher impact parameter, therefore a larger star and a bigger planet. However, this is partly compensated by the fact that these datasets also favour a slightly shallower transit, therefore a smaller planet. Altogether, the uncertainty on the planetary radius is small enough for useful comparison with models.

We confirm that HD 80606 is not a "bloated" hot Jupiter, as discussed in M09, and has a size compatible with current models for irradiated gas giants. Given its large mass, a planet like HD 80606b would require more additional energy that Jupitermass planets to be inflated to a higher radius. However, two other exoplanets with similar masses, CoRoT-Exo-2b $\left(3.3 \mathrm{M}_{\mathrm{J}}\right)$ and OGLE2-TR-L9b $\left(4.5 M_{\mathrm{J}}\right)$ have radii around $1.5 R_{\mathrm{J}}$, showing that such inflated sizes are indeed possible for these heavy planets. The two other known transiting planets in the 3-5 $M_{\mathrm{J}}$ mass 


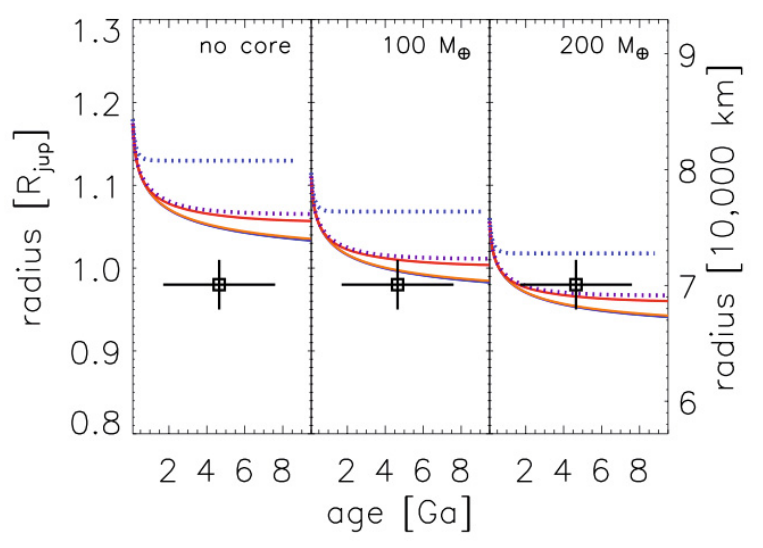

Fig. 9. Evolution of the planetary radius according to models for HD 80606b. Left: without heavy-element core. Middle: with a $100 M_{\oplus}$ core. Right: with a $200 M_{\oplus}$ core. From bottom to top, the lines correspond to different assumption on the physics of the evolution models used to match the sizes of transiting planets: blue: standard models with no extra heating; orange: dissipating $0.5 \%$ of incoming stellar heat flux energy in the interior of the plane; red: opacities increased by a factor 30. The dotted (blue) lines correspond to models with an extra luminosity dissipated at the center of the planet $L_{\text {tide }}=10^{26}$ and $10^{27} \mathrm{erg} \mathrm{s}^{-1}$, respectively.

range, HD $17156 \mathrm{~b}$ and WASP-10b, have radii of 1.0 and $1.1 R_{\mathrm{J}}$ respectively.

The size of these planets is conspicuously correlated with the amount of flux received from the parent star. CoRoT-Exo-2b and OGLE2-TR-L9b follow close orbits (1.7 and 2.4-day periods), while HD 17156b and HD 80606b have the widest orbits of known transiting planets, with WASP-10b being an intermediate case. The observed correlation between size and incident flux for transiting gas giants may therefore extend to several Jupiter masses, which provides another benchmark that any successful explanation of anomalous exoplanet radius must meet.

Figure 9 shows the evolution of the radius of HD 80606b according to models described in Guillot et al. (2006), with the position of our reference solution indicated. The different panels show the radius evolution of a planet with a solar-composition envelope (containing about $25 M_{\oplus}$ in heavy elements), but either without core (left panel), with a $100 M_{\oplus}$ core (middle panel) or with a $200 M_{\oplus}$ core (right panel). Clearly, although uncertainties in the distribution of heavy elements and equations of state have to be taken into account (e.g. Baraffe et al. 2008), our models point towards the existence of a large mass in heavy elements (at least $60 M_{\oplus}$ ) present in the planet. All possibilities above 60 and $200 M_{\oplus}$ are consistent with the measured radius. Solutions with even larger cores are possible, but we regard them as unlikely given that realistic critical core masses are smaller than $100 M_{\oplus}$ (Ikoma et al. 2006) and planets with masses larger than Jupiter tend to scatter planetesimals much more efficiently than they accrete them (e.g. Guillot \& Gladman 2000).

Given that HD80606 is metal-rich, this large inferred core mass is consistent with a correlation between heavy elements in the star and in the planet (Guillot et al. 2006; Burrows et al. 2007; Guillot 2008). It should be noted that if the planet had the same composition as the star, its total mass of heavy elements should be of order $50 M_{\oplus}$, clearly smaller than the estimates that are derived here.

Interestingly, using the concept of a maximum mass in heavy elements in the planet yields an upper limit on the rate of tidal
Table 3. Predicted ephemerides for the next transit events.

\begin{tabular}{lll}
\hline \hline Date & Ingress [BJD] & Egress [BJD] \\
\hline 5 June 2009 & $2454987.53 \pm 0.05$ & $2454987.974 \pm 0.005$ \\
24 September 2009 & $2455098.96 \pm 0.05$ & $2455099.409 \pm 0.005$ \\
13-14 January 2010 & $2455210.40 \pm 0.05$ & $2455210.844 \pm 0.006$ \\
5 May 2010 & $2455321.84 \pm 0.05$ & $2455322.281 \pm 0.006$ \\
24-25 August 2010 & $2455433.27 \pm 0.05$ & $2455433.717 \pm 0.006$ \\
14 December 2010 & $2455544.71 \pm 0.05$ & $2455545.152 \pm 0.007$ \\
4-5 April 2011 & $2455656.14 \pm 0.05$ & $2455656.588 \pm 0.008$ \\
\hline
\end{tabular}

dissipation that it may undergo. As shown by the dotted lines in Fig. 9, a maximum rate of dissipation compatible with the observations and models with core of at most $200 M_{\oplus}$ is of order $10^{27} \mathrm{erg} \mathrm{s}^{-1}$. While this may seem large, it is several times smaller that the dissipation due to tides for this planet if it has been brought in by a Kozai mechanism (Wu \& Murray 2003), estimated by $G M_{*} M_{\mathrm{p}} /\left(a_{\mathrm{p}} \tau_{\text {migration }}\right) \approx 5 \times 10^{27} \mathrm{erg} \mathrm{s}^{-1}$ (where we used $a_{\mathrm{p}}$ as the present semi-major axis of the planet, and $\tau_{\text {migration }} \approx 1 \mathrm{Ga}$ from WM03). Several possibilities exist: (i) The planet may have a tidal Q larger than the $3 \times 10^{5}$ assumed by WM03 so that it would have migrated on a longer timescale; (ii) Heat dissipation may occur in a shallow region close to the atmosphere of the planet and be reradiated quickly after the approach to the star; (iii) The planet may have a core that is much larger than envisioned in the present study. Except in the last case, our calculations indicate that the planet's intrinsic effective temperature should be of order 200 to $300 \mathrm{~K}$, much smaller than the 600 to $700 \mathrm{~K}$ envisioned by Wu \& Murray (2003) and Laughlin et al. (2009).

\subsection{Future observations}

Given the brightness of the host star and its peculiar orbit, HD 80606 is likely to remain an important target for further studies. Table 3 shows the predicted times of ingress and egress for the next transit events according to our reference solution and $68 \%$ central confidence intervals.

When the transit duration will be measured by observation of future transit events, the corresponding value of the most probable impact parameter and planetary radius can be read directly off Fig. 7.

\section{Conclusion}

Our analysis of combined photometric and spectroscopic observations of HD 80606 have led to new estimates of the stellar and planetary parameters of the system. These values allow relatively tight constraints on the planet's composition, with a likely mass in heavy elements between 60 and $200 M_{\oplus}$, which imply a rather efficient accretion of planetesimals most probably during the planet's formation and early evolution.

We have also shown that the planet's orbit is probably not aligned with the star's spin: this strengthens the argument that the planet may have migrated inwards through a Kozai mechanism and thus acquired its large eccentricity (Wu \& Murray 2003).

Our analysis can be readily put to the test with future observations of planetary transits, as we predict a total duration of the eclipse of $T_{1-4}=11.9 \pm 1.3 \mathrm{~h}$. Furthermore, the analysis of the planet's primary and secondary transits, and of the planet's irradiation in the infrared when far from the star will also be extremely fruitful to understand how tidal energy is dissipated: we 
predict that the intrinsic effective temperature of the planet is between 200 and $300 \mathrm{~K}$, smaller than the zero-albedo mean equilibrium temperature due to stellar irradiation, $400 \mathrm{~K}$. In contrast, larger effective temperatures $(\sim 700 \mathrm{~K})$ have been hypothetized, due to efficient dissipation of heat in the planet (Laughlin et al. 2009).

Acknowledgements. Financial support for the SOPHIE Consortium from the "Programme national de planétologie" (PNP) of CNRS/INSU, France, and from the Swiss National Science Foundation (FNSRS) are gratefully acknowledged. We also acknowledge support from the UK Science and Technology Facilities Council, the French CNRS and Fundação para a Ciência e a Tecnologia, Portugal. The MEarth team gratefully acknowledges funding from the David and Lucile Packard Fellowship for Science and Engineering, and support from the US National Science Foundation under grant number AST-0807690.

\section{References}

Baraffe, I., Chabrier, G., \& Barman, T. 2008, A\&A, 482, 315

Burrows, A., Hubeny, I., Budaj, J., et al. 2007, ApJ, 661, 502

Butler, R. P., Wright, J. T., Marcy, G. W., et al. 2006, ApJ, 646, 505

Collier Cameron, A., Wilson, D. M., West, R. G., et al. 2007, MNRAS, 380, 1230

Fabrycky, D. C., \& Winn, J. N. 2009, ArXiv e-prints

Fischer, D. A., \& Valenti, J. 2005, ApJ, 622, 1102

Fossey, S. J., Waldman, I. P., \& Kipping, D. M. 2009, ArXiv e-prints

Garcia-Melendo, E., \& McCullough, P. R. 2009, ArXiv e-prints
Girardi, L., Bertelli, G., Bressan, A., et al. 2002, A\&A, 391, 195

Guillot, T. 2008, Phys. Scrip. Vol. T, 130, 014023

Guillot, T., \& Gladman, B. 2000, in Late Planetesimal Delivery and the Composition of Giant Planets, ed. Garzón, G., Eiroa, C., de Winter, D., \& Mahoney T. J., Disks, Planetesimals, and Planets Vol. 219 of ASP Conf. Ser., 475

Guillot, T., Santos, N. C., Pont, F., et al. 2006, A\&A, 453, L21

Hébrard, G., Bouchy, F., Pont, F., et al. 2008, A\&A, 488, 763

Ikoma, M., Guillot, T., Genda, H., Tanigawa, T., \& Ida, S. 2006, ApJ, 650, 1150

Irwin, J., Irwin, M., Aigrain, S., et al. 2007, MNRAS, 375, 1449

Irwin, J., Charbonneau, D., Nutzman, P., et al. 2009, in The MEarth project:

searching for transiting habitable super-Earths around nearby M dwarfs, IAU Symp., 253 of IAU Symp., 37

Laughlin, G., Deming, D., Langton, J., et al. 2009, Nature, 457, 562

Mandel, K., \& Agol, E. 2002, ApJ, 580, L171

Moutou, C., Hebrard, G., Bouchy, F., et al. 2009, ArXiv e-prints

Naef, D., Latham, D. W., Mayor, M., et al. 2001, A\&A, 375, L27

Nutzman, P., \& Charbonneau, D. 2008, PASP, 120, 317

Ohta, Y., Taruya, A., \& Suto, Y. 2005, ApJ, 622, 1118

Pont, F., \& Eyer, L. 2004, MNRAS, 351, 487

Pont, F., Zucker, S., \& Queloz, D. 2006, MNRAS, 373, 231

Pont, F., Holman, M., \& Sasselov, D. 2009, Transiting Planets. IAU Symp. 253 (Cambridge University Press)

Ribas, I., \& Miralda-Escudé, J. 2007, A\&A, 464, 779

Ryan, P., \& Sandler, D. 1998, PASP, 110, 1235

Winn, J. N., Johnson, J. A., Fabrycky, D., et al. 2009, ArXiv e-prints

Wittenmyer, R. A., Endl, M., Cochran, W. D., Levison, H. F., \& Henry, G. W. 2009, ApJS, 182, 97

Wu, Y., \& Murray, N. 2003, ApJ, 589, 605

Young A. T., 1967, AJ, 72, 747 